\title{
Neighbor-friendly autonomous power control in wireless heterogeneous networks
}

\author{
Rodolfo Torrea-Duran ${ }^{1 *}$, Paschalis Tsiaflakis², Luc Vandendorpe ${ }^{3}$ and Marc Moonen ${ }^{1}$
}

\begin{abstract}
The widespread deployment of base stations constitutes a promising solution to cope with the ever-increasing wireless data rate demands. However, it also increases the interference levels, especially at the cell-edge. Most interference management techniques assume coordination between base stations, which involves undesired overhead and delays. To solve this problem, we propose a neighbor-friendly autonomous algorithm for power control in wireless heterogeneous networks that protects victim users from neighboring cells through a penalty factor in the power allocation level. We refer to this algorithm as neighbor-friendly iterative waterfilling (NF-IWF). In addition, we propose a low-complexity closed-form version that fixes the penalty factor by assuming a linear approximation of the victim user data rate. In high interference conditions, it can achieve a victim user data rate increase by a factor of 3.5 compared to IWF, 15 compared to soft frequency reuse (SFR), and 60 compared to equal power allocation (EPA) with a marginal decrease of the primary user data rate.
\end{abstract}

Keywords: Interference management; Heterogeneous networks; Autonomous power control

\section{Introduction}

The boost of novel wireless applications is producing an ever-increasing demand for larger data rates.

Furthermore, the massive market penetration of smartphones adds more pressure to network operators, mobile manufacturers, and standardization groups to satisfy the demands of an ever-larger number of users.

Improvements from a physical layer perspective have shown a low potential to deal with these demands. For instance, considering new coding techniques, link adaptation, and larger bandwidths, from 1950 to 2000, the network capacity gains have increased by a factor of 5 from the first, a factor of 5 from the second, and a factor of 15 from the third.

Surprisingly, just from the deployment of more (smaller) cells, the network capacity gains have increased by an overwhelming factor of 2,700 [1].

Femtocells offer the easiest and most cost-effective way to increase the current cell deployment [2].

These are low-power base stations mainly for indoor usage with a coverage of tens of meters, compared to a

*Correspondence: Rodolfo.TorreaDuran@esat.kuleuven.be

1 STADIUS Center for Dynamical Systems, Signal Processing and Data Analytics, Kasteelpark Arenberg 10, 3000 Leuven, Belgium

Full list of author information is available at the end of the article few kilometers of high-power base stations, or macrocells. The coexistence of different types of cells in the same area is referred to as a heterogeneous network.

Most urban areas are already fully covered by macrocells. Hence, the addition of more cells in these areas creates serious inter-cell interference problems in both uplink and downlink transmissions, especially in closedaccess mode. In this paper, we focus on the downlink transmission. Since femtocells are likely to be deployed in an unplanned manner by end-users and not by network operators, this problem is only going to grow in the coming years $[3,4]$.

Base stations play a crucial role in dealing with an inter-cell interference. The power and time-frequency resources they allocate to users within the cell have an impact on users from neighboring cells. The impact is especially high for users located at the cell-edge.

Therefore, the implementation of inter-cell interference coordination (ICIC) techniques is of paramount importance. Nevertheless, most of the ICIC techniques allocate separate resources to neighboring cells, i.e., they exploit resource orthogonalization [5-10]. This is usually done in the frequency domain or in the time domain, while variable power levels are rarely exploited. Thus, in this paper, we focus on and advocate for power control schemes that 
control an inter-cell interference by sharing (rather than orthogonalizing) resources.

Power control techniques implemented in current wireless networks such as LTE focus on resource orthogonalization between cells. A typical example is soft frequency reuse (SFR) [11], in which the total bandwidth of each cell is divided into two non-overlapping frequency bands, one for center users (primary users in our case) and one for cell-edge users (victim users in our case). The center user band can be reused in all the cells, while the cell-edge user band is non-overlapping between neighboring cells, as can be seen in Figure 1. The transmit power level is constant in each band but larger for the cell-edge user band to compensate for the performance degradation.

This approach offers a simple way to deal with frequency and power allocation jointly without interference but with the disadvantage of a fixed allocation of frequency bands and transmit powers. The optimization of SFR is usually done by adapting parameters $\alpha$ and $\beta$, which represent the total power fraction and total bandwidth fraction of the center user band, respectively [12]. However, this can only be achieved through network coordination.

To support coordination between base stations, a dedicated backhaul link is required. In LTE, the X2 interface between macrocells helps to configure dynamically the frequency bands and power levels [13-17].

However, this interface is not yet standardized between macro- and femtocells in early versions of LTE release $[11,18,19]$. Even with an interface available, the dynamics of the wireless channel and the variability in the number

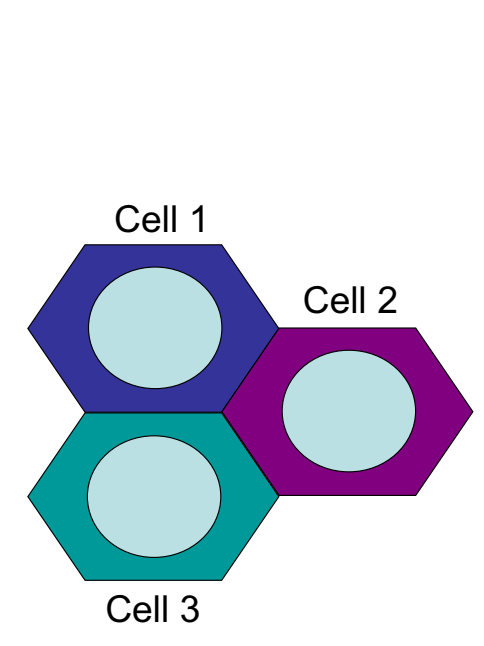

of users attached to a cell, users' location, and interference conditions, this coordination constitutes a significant information overhead resulting in large delays.

An alternative approach is that each base station optimizes its own resource allocation without any information exchange. We refer to it as an autonomous algorithm.

Two well-known autonomous power control algorithms for OFDM networks are equal power allocation (EPA) and iterative waterfilling (IWF) [20]. In EPA, the total transmit power is allocated equally in all available subcarriers. In IWF, each base station maximizes its own data rate in a greedy way by allocating more power to those subcarriers with the best channel to interference and noise ratio (CINR), without considering the interference caused to victim users from neighboring cells.

Non-greedy autonomous power control algorithms have been studied in the context of digital subscriber line (DSL) networks.

In [21] and [22], low-complexity autonomous power control algorithms for DSL, called ASB and ASB-2 are presented, which allow a non-orthogonalized share of resources. The concept of a protected reference line (or reference user) is introduced in these papers as a statistical average of all victim lines suffering interference. However, the implementation of these algorithms in practical wireless networks imposes a challenge given the multi-user scheduling and non-stationarity of the wireless channel.

A first attempt was made in [23] to apply the previous concept to a wireless network where the user suffering the strongest interference from the neighboring cells is selected as the reference user. However, this scheme is

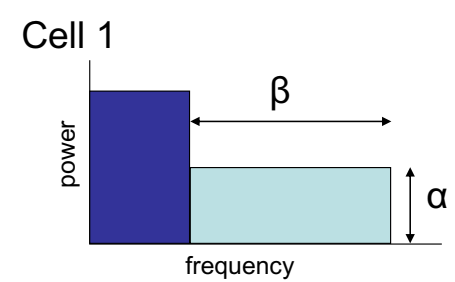

Cell 2

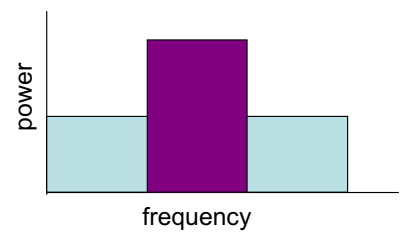

Cell 3

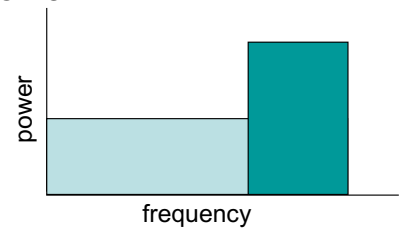

Figure 1 Soft frequency reuse. 
not autonomous since it needs periodical information exchange between base stations to adapt to the timevarying reference user channel characteristics.

To tackle the mentioned problems, we propose in this paper a neighbor-friendly autonomous algorithm for power control in wireless heterogeneous networks. It protects victim users within a certain distance from the base station through a frequency-dependent penalty factor in the power allocation level. The level of protection can be tuned to provide individualized quality of service (QoS). We refer to this algorithm as neighbor-friendly iterative waterfilling (NF-IWF). Additionally, we propose a lowcomplexity closed-form version that fixes the penalty factor by assuming a linear approximation of the data rate of the victim users.

We can summarize the main contributions of the paper as follows:

1. A power control algorithm that protects victim users from neighboring cells located at a distance from the base station without any coordination between base stations.

2. A practical way to tune the protection to victim users from neighboring cells.

3. A practical way to obtain the channel information of victim users attached to neighboring cells without communication between base stations.

4. A low-complexity closed-form version of the previous algorithm by assuming a linear approximation of the data rate of the victim users.

5. The exploitation of frequency and time correlation of the wireless channel to further reduce the complexity of the algorithm.

\section{IWF-based power control}

Autonomous algorithms do not rely on information between base stations, they only exploit locally available (and a priori known) information about the environment such as direct channel gains, received interference, and noise.

IWF exploits this information to maximize the data rate in each cell:

$$
\begin{array}{cl}
\underset{s_{k}^{c} \forall k}{\operatorname{maximize}} & R^{c} \\
\text { s.t. } & \sum s_{k}^{c} \leq P^{c, \text { tot }} \\
& 0 \leq s_{k}^{c} \leq s_{k}^{c, \text { mask }} \forall k \in \mathcal{K}
\end{array}
$$

with

$$
R^{c}=f_{s} \sum_{k \in \mathcal{K}} b_{k}^{c}=f_{s} \sum_{k \in \mathcal{K}} \log _{2}\left(1+\frac{1}{\Gamma} \frac{\left|h_{k}^{c}\right|^{2} s_{k}^{c}}{\sum_{\substack{\bar{c} \neq c \\ \bar{c} \in \mathcal{C}}}\left|h_{k}^{\bar{c}}\right|^{2} s_{k}^{\bar{c}}+\sigma_{k}^{c}}\right)
$$

where $R^{c}$ is the data rate of all users in cell $c ; f_{s}$ is the symbol rate; $b_{k}^{c}, h_{k}^{c}, \sigma_{k}^{c}, s_{k}^{c}$, and $s_{k}^{c \text {,mask }}$ are the bit loading for a standard interference channel model, the channel transfer function, the noise power, the base station transmit power, and the spectral emission mask constraints on subcarrier $k$ in cell $c$, respectively; $h_{k}^{\bar{c}}$ and $s_{k}^{\bar{c}}$ are the channel transfer function and transmit power on subcarrier $k$ from the interfering cell $\bar{c}$, which are both assumed to be known as they affect users in cell $c$. We call $h_{k}^{c}$ the direct channel and $h_{k}^{\bar{c}}$ the interfering channel of the users attached to cell $c$. The parameters $\mathcal{C}$ and $\mathcal{K}$ are the set of available cells and subcarriers, respectively, and $P^{c, \text { tot }}$ is the total power budget in cell $c$. A given subcarrier can only be allocated to one user in cell $c$, but it can also be allocated to a user from a neighboring cell resulting in an inter-cell interference. The allocation of subcarriers to users in cell $c$ can be done prior to the power allocation strategies described in this paper (based, for example, on instantaneous channel conditions or iteratively with the power allocation). However, our focus is only on the power allocation. A joint autonomous power and subcarrier allocation algorithm is nevertheless an interesting scenario that we will study for future work. The parameter $\Gamma$ denotes the signal-to-noise ratio (SNR) gap to capacity, which depends on the desired bit error rate (BER), the coding gain, and the noise margin. We will assume it to be equal to 1 without loss of generality.

It can be shown, using the corresponding Karush-KuhnTucker (KKT) conditions, that the transmit powers have a closed-form solution as follows

$$
s_{k}^{c}=\left[\frac{f_{s}}{\log (2) \lambda_{c}}-\frac{\sum_{\bar{c} \neq c} \Gamma\left|h_{k}^{\bar{c}}\right|^{2} s_{k}^{\bar{c}}+\Gamma \sigma_{k}^{c}}{\left|h_{k}^{c}\right|^{2}}\right]_{0}^{s_{k}^{c \text { mask }}}
$$

where $[x]_{a}^{b}=\max (a, \min (x, b))$ and $\lambda_{c}$ is the Lagrange multiplier that should be updated (e.g., with bisection) to satisfy the corresponding total power constraint $P^{c \text {,tot }}$.

To analyze the benefit of exploiting locally available information, we consider a network with 5 macrocells evenly distributed over a $30 \times 30 \mathrm{~km}$ area, each with a $43 \mathrm{dBm}$ total transmit power and $5 \mathrm{MHz}$ bandwidth. As a channel model, we use the 3GPP spatial channel model (SCM [24]) with suburban macro environment and a distance $(d)$-dependent path loss of $31.5+35 \log _{10}(d[m])$. We show in Figure 2 the difference between the data rates obtained using IWF and EPA of a user moving along every possible location on this area. The results can be seen in a tri-dimensional plot where the $x$ and $y$ axes correspond to the area, and the $z$ axis corresponds to the data rate difference between IWF and EPA. A maximum difference of $400 \mathrm{Mbps}$ can be observed, interestingly, at the cell-edge of neighboring cells. 


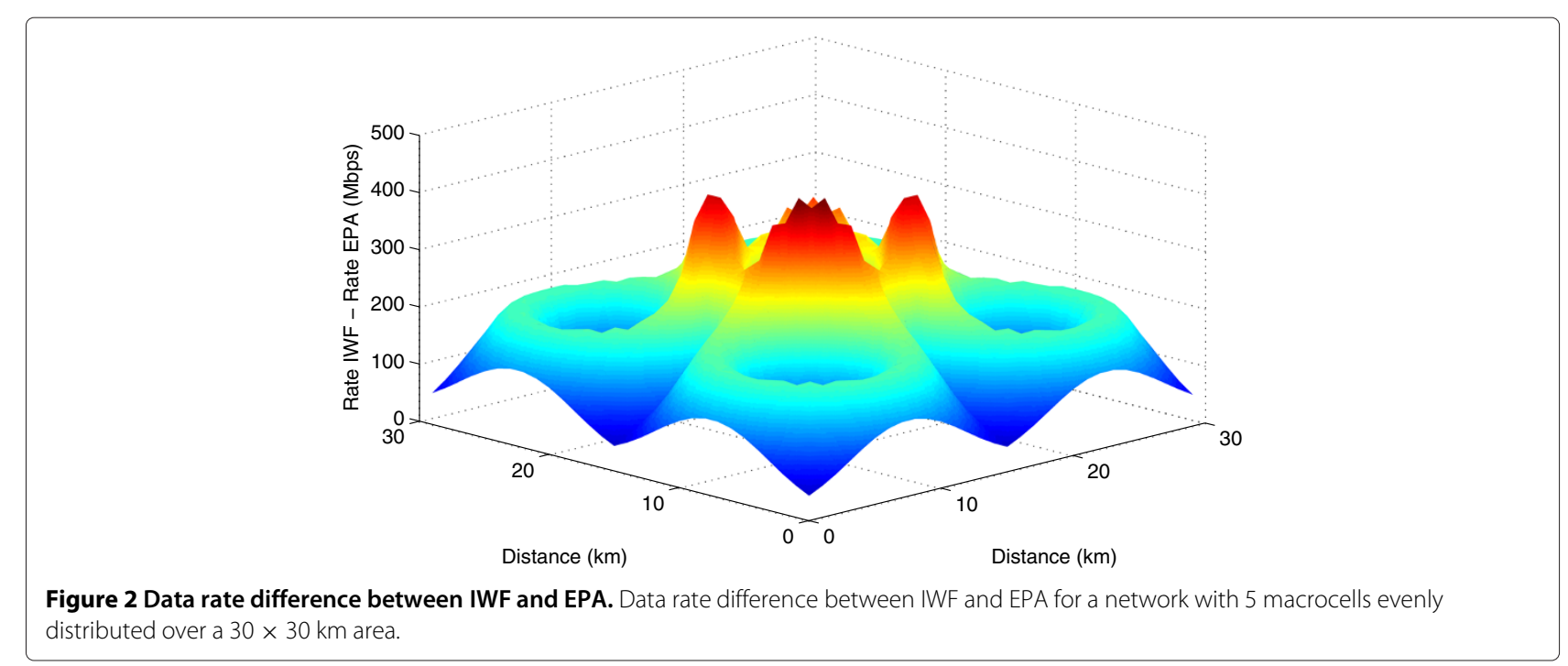

This scenario becomes more critical when femtocells are deployed. We now introduce into the previous network topology 20 femtocells randomly distributed (i.e., deployed in an unplanned manner), each with a $15 \mathrm{dBm}$ total transmit power. The exact location of each base station can be seen in Figure 3. We compute again the difference between the data rates obtained using IWF and EPA of a user moving along every possible location. The results are shown in Figure 4. The femtocells' deployment results in more severe interference problems, especially at the cell-edges. Remarkably, a maximum difference of 1,250 Mbps can be observed at the cell-edge of multiple interfering cells (macro- and femtocells). This again shows the potential gains exploiting locally available information.
The advantage of IWF is its simplicity, its closed-form solution, and the fact that it does not need any information exchange between base stations. However, each cell maximizes its own data rate in a greedy fashion by allocating power especially to those subcarriers with the best CINR, without considering the interference caused to victim users from neighboring cells.

\section{Neighbor-friendly autonomous power control Victim users protection with NF-IWF}

Our aim is therefore to design a neighbor-friendly approach that, without any information exchange in the network, limits this damage.

Using the concept of a protected reference user, we formulate optimization problem (1) as the weighted sum

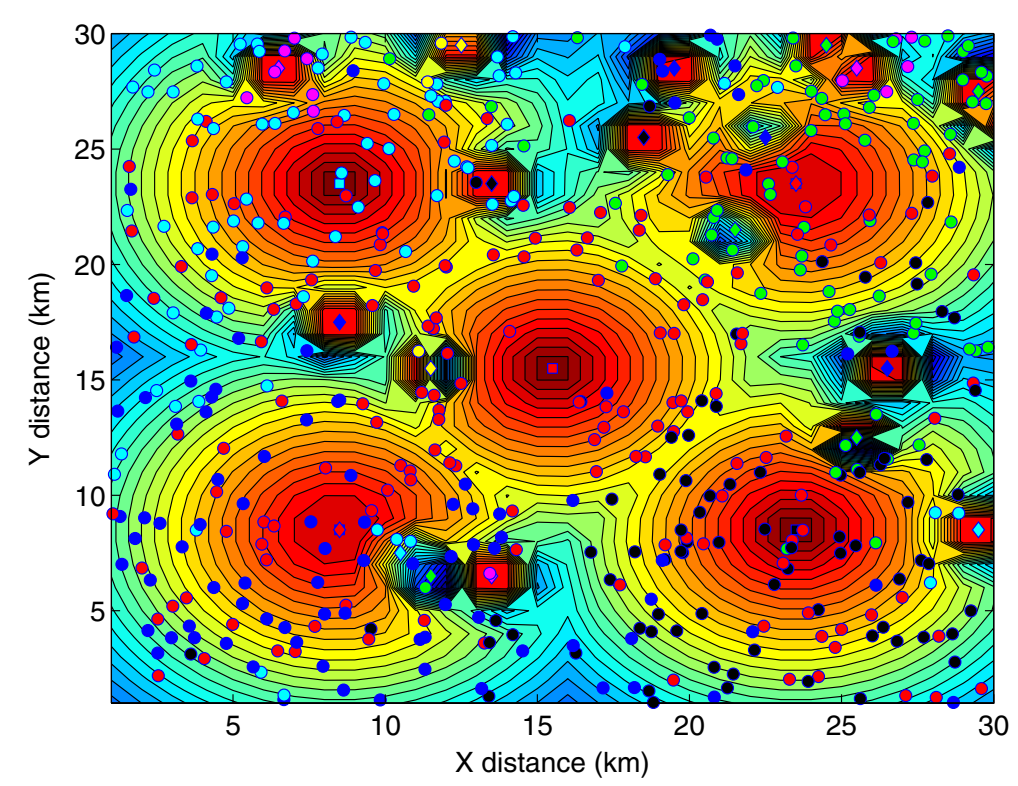

Figure 3 Network with 5 macrocells, 20 femtocells, and 500 users. 


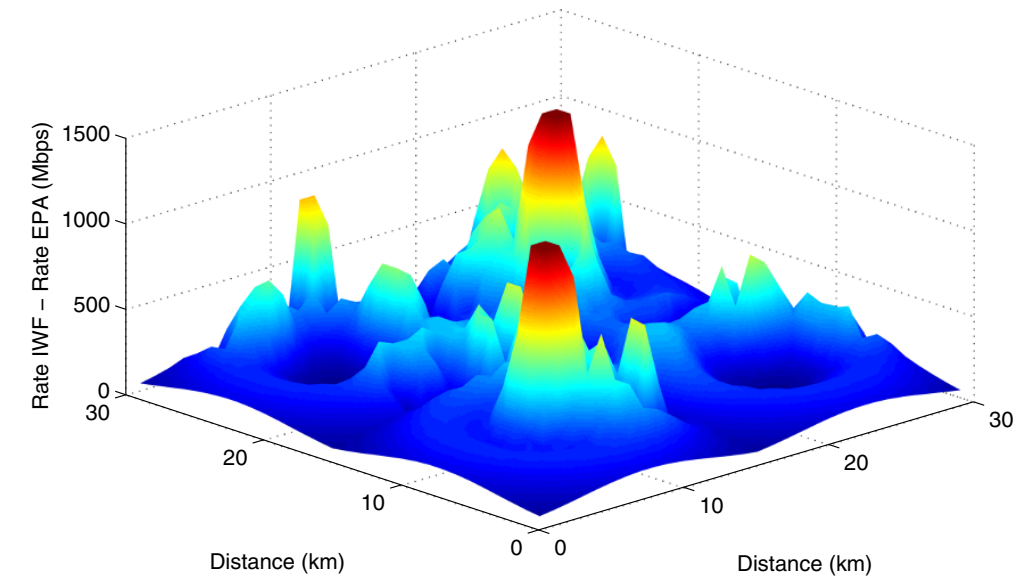

Figure 4 Data rate difference between IWF and EPA. Data rate difference between IWF and EPA for a network with 5 macrocells evenly distributed and 20 femtocells randomly distributed over a $30 \times 30 \mathrm{~km}$ area.

of the data rate of users attached to cell $c$, or primary users, denoted as $R^{c}$ and the data rate of victim users attached to one neighboring cell, denoted as $R^{v c}$. Both cells share the set of subcarriers $\mathcal{K}$. Adding extra optimization terms for other neighboring cells only brings minimal performance at a high-complexity cost since most of the per-subcarrier interference comes from one base station [23]. However, other neighboring cells can be considered if they share a different set of subcarriers with cell $c$. Again, the subcarrier allocation to users is assumed to be done prior to the power allocation.

$$
\begin{array}{cl}
\underset{s_{k}^{c} \forall k}{\operatorname{maximize}} & w^{c} R^{c}+w^{\nu c} R^{v c} \\
\text { s.t. } & \sum_{k} s_{k}^{c} \leq P^{c, \text { tot }} \\
& 0 \leq s_{k}^{c} \leq s_{k}^{c, \text { mask }} \forall k \in \mathcal{K}
\end{array}
$$

with

$$
R^{\nu c}=f_{s} \sum_{k \in \mathcal{K}} b_{k}^{\nu c}=f_{s} \sum_{k \in \mathcal{K}} \log _{2}\left(1+\frac{1}{\Gamma} \frac{\left|h_{k}^{\nu c}\right|^{2} s_{k}^{\nu c}}{\left|h_{k}^{\nu c,}\right|^{2} s_{k}^{c}+\sigma_{k}^{\nu c}}\right)
$$

where $b_{k}^{\nu c}, h_{k}^{v c}, s_{k}^{\nu c}$, and $\sigma_{k}^{\nu c}$ are the bit loading, the direct channel, the transmit power, and the noise power on subcarrier $k$ of the victim user, respectively, and $h_{k}^{v c, c}$ is the interfering channel on subcarrier $k$ from cell $c$ to a victim user. $w^{c}$ and $w^{v c}$ are the weights of the primary users and the victim users, respectively. We assume $w^{c}$ equal for all primary users and $w^{v c}$ equal for all victim users. We consider that $w^{c}=1-w^{v c}$, which represents a trade-off between protecting victim users of a neighboring cell at the cost of degrading the data rate of primary users. In practice, these weights can be chosen based on upper layer information such as queue length or quality of service requirements. Setting $w^{v c}=0$ shifts to a greedy algorithm like IWF, which can be useful when interference between neighboring cells is negligible.

Applying the KKT stationarity condition to problem (4) leads to

$$
\begin{aligned}
\forall k & : \frac{w^{c} f_{s}\left|h_{k}^{c}\right|^{2}}{\log (2)\left(\left|h_{k}^{c}\right|^{2} s_{k}^{c}+\sum_{\bar{c} \neq c} \Gamma\left|h_{k}^{\bar{c}}\right|^{2} s_{k}^{\bar{c}}+\Gamma \sigma_{k}^{c}\right)} \\
& -\frac{w^{v c} f_{s}\left|h_{k}^{v c}\right|^{2} s_{k}^{v c}\left|h_{k}^{v c, c}\right|^{2}}{\log (2)\left(\Gamma\left|h_{k}^{v c, c}\right|{ }^{2} s_{k}^{c}+\Gamma \sigma_{k}^{\nu c}\right)\left(\left|h_{k}^{v c}\right|^{2} s_{k}^{v c}+\Gamma\left|h_{k}^{v c, c}\right|^{2} s_{k}^{c}+\Gamma \sigma_{k}^{v c}\right)} \\
& -\lambda_{c}=0 .
\end{aligned}
$$

By taking into account the KKT complementarity conditions of (4), $s_{k}^{c}$ from the first term of Equation 6 can be isolated to obtain:

$$
s_{k}^{c}=\left[\frac{\frac{w^{c} f_{s}}{\log (2)}}{\lambda_{c}+P_{k}^{N F, c}}-\frac{\sum_{\bar{c} \neq c} \Gamma\left|h_{k}^{\bar{c}}\right|^{2} s_{k}^{\bar{c}}+\Gamma \sigma_{k}^{c}}{\left|h_{k}^{c}\right|^{2}}\right]_{0}^{c_{k}^{c \text { mask }}}
$$

where $P_{k}^{N F, c}$ is referred to as the penalty factor, defined as

$$
\begin{aligned}
& P_{k}^{N F, c} \\
& =\frac{w^{\nu c} f_{s}\left|h_{k}^{\nu c}\right|^{2} s_{k}^{\nu c} \Gamma\left|h_{k}^{v c, c}\right|^{2}}{\log (2)\left(\Gamma\left|h_{k}^{\nu c, c}\right|^{2} s_{k}^{c}+\Gamma \sigma_{k}^{\nu c}\right)\left(\left|h_{k}^{v c}\right|^{2} s_{k}^{\nu c}+\Gamma\left|h_{k}^{\nu c, c}\right|^{2} s_{k}^{c}+\Gamma \sigma_{k}^{\nu c}\right)} .
\end{aligned}
$$

Note the similarity with the IWF solution. The first term in Equation 7 corresponds to a power level with per-subcarrier penalty factor $P_{k}^{N F, c}$ that reduces the interference to victim users from a neighboring cell. Setting $P_{k}^{N F, c}$ to zero will reduce to the IWF algorithm. However, in contrast to Equation 3, this is a fixed-point equation as $P_{k}^{N F, c}$ depends on $s_{k}^{c}$. 
Problem 4 is a nonconvex function for which a duality gap exists to the optimal solution. However, as the number of subcarriers increase, this duality gap becomes zero and it can be solved via bisection [25].

By adding to Equation 7 a bisection search on the Lagrange multiplier to satisfy the total cell power constraint, we obtain Algorithm 1, which we refer to as NF-IWF. The parameter $\delta$ indicates the accuracy of the total power constraint, $\gamma$ indicates the stopping criterion of the bisection search on $\lambda_{c}$ in the case of an inactive total power constraint, and $\Lambda^{\max }$ is the maximum value for $\lambda_{c}$.

The transmit powers of the neighboring cells $s_{k}^{\nu c}$ are assumed as an EPA without performance degradation as observed in later sections.

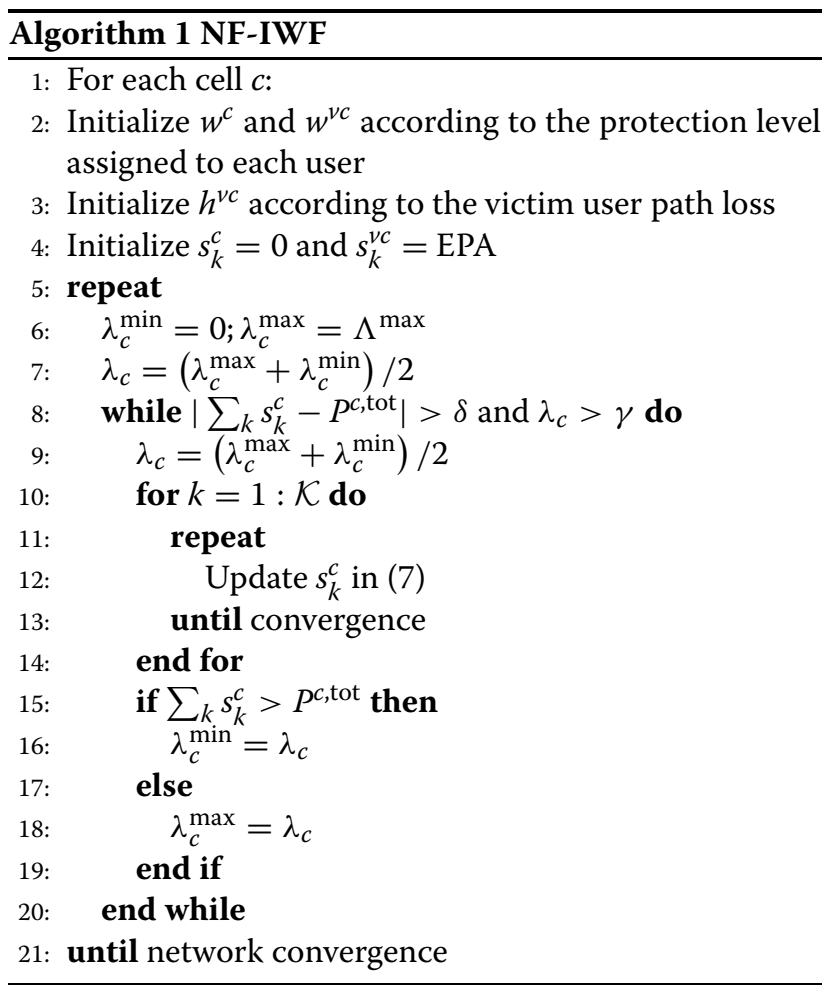

Contrary to most ICIC techniques that orthogonalize resources, an advantage of NF-IWF is that it allows subcarriers to be shared between users as long as adequate power levels are used. This can be seen in Figure 5. Interestingly, NF-IWF allocates more power to those subcarriers less used for transmission by the interfering base station, which uses IWF. Still, some subcarriers are shared by both base stations.

The challenge of implementing NF-IWF in a wireless network resides on obtaining the parameters in Equation 8 in an autonomous way. For example, $h_{k}^{\nu c, c}$ can be obtained from the channel feedback of victim users when scanning pilot signals of a neighboring cell for a potential handover $[26,27]$. However, the direct channel $h_{k}^{v c}$ can only be known from the information received from other base stations.

Therefore, we propose in the 'Estimation of the victim user channel' section a novel approach to estimate $h_{k}^{v c}$ based on the distance from the base station to the cell-edge.

\section{Estimation of the victim user channel}

Full knowledge of $h_{k}^{v c}$ is unfeasible in an autonomous fashion.

Nevertheless, the path loss, i.e., the average channel gain over all the allocated subcarriers, is easier to obtain because it mainly depends on the distance to the base station. Since the signal strength coming from two neighboring base stations can be considered equal at the cell-edge (this is how the cell-edge is typically defined), the path loss from each base station to the cell-edge can be known. This can be exploited to approximate the direct channel of any victim user $\left(h_{k}^{v c}\right)$ by using the path loss from the base station to the cell-edge. Therefore, $\tilde{h}^{v c}$ is assumed to be an average over all the available subcarriers such that any user located at a distance $d$ from the base station would present the same average channel and experience the same path loss given by $31.5+35 \log _{10}(d[\mathrm{~m}])$ [24]. This results in a constant value for $h_{k}^{v c}$ along all the set of subcarriers $\mathcal{K}$, i.e., $h_{k}^{v c}=\tilde{h}^{v c} \forall k \in \mathcal{K}$ where $\tilde{h}^{v c}=\frac{1}{\mathcal{K}} \sum_{k=1}^{\mathcal{K}} h_{k}^{c}$. Even though an irregular propagation channel (i.e., with shadowing) might affect each victim user differently, we will see later that $\tilde{h}^{v c}$ is a good approximation for the direct channel of all potential victim users if $h_{k}^{v c, c}$ of each user is known.

To estimate $d$ in practice, we can use the information available at the base station on the channel estimated by new users entering the cell [28] or predefined by the manufacturer. As we will see later, an exact definition of $d$ is not necessary for improved performance.

Furthermore, this information does not need to be updated regularly (since the cell-edge will only be modified when a new base station is deployed in the neighborhood).

Despite its simplicity, this model provides an accurate estimation of the victim users channel characteristics. To analyze the sensitivity of this model, we consider two users in a high interference case 1 (where the victim user is severely interfered by cell $c$ ) and a low interference case 2 (where the victim user is at the cell-edge) as reconstructed in Figure 6 and Figure 7, respectively. MBS stands for macro base station, and FBS stands for femto base station. The color regions indicate the signal strength in the direct channel to the closest base station, and the user color indicates the base station to which the user is attached.

We assume that there is no handover, like in a closedaccess base station. 


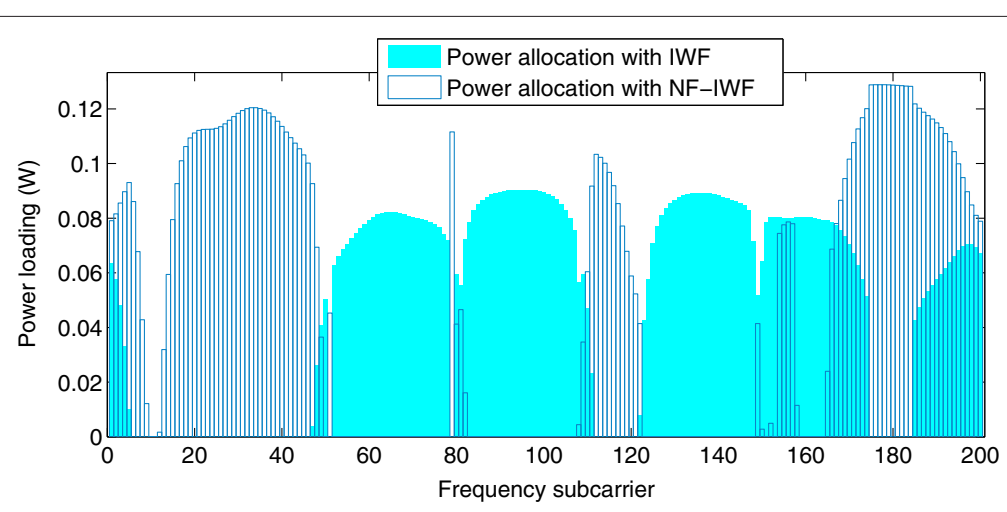

Figure 5 Transmit power allocation from two neighboring base stations to two cell-edge users over 200 subcarriers. Each base station is transmitting to a cell-edge user and interfering with the other base station. One base station uses NF-IWF and the other uses IWF.

Figure 8 computes the normalized data rate of both users for cases 1 and 2 by evaluating $\tilde{h}^{v c}$ at different values of $d$, but keeping the users' positions as in Figure 5 and Figure 6. The distance $d$ is then varied between 0 and the actual distance between base stations and it is normalized such that $d_{\text {norm }}=0$ means a radius of zero and $d_{\text {norm }}=1$ means a radius equal to the distance between both base stations $\left(d_{\mathrm{BS}}\right)$. The smaller the path loss distance, the more protection to the victim user at the cost of the primary user data rate.

At $d_{\text {norm }}=0.5$, i.e., between the two base stations, we have the highest normalized data rate of the victim user for a primary user data rate of $20 \%$ in case 1 and $50 \%$ in case 2 . This is because in case 1 , the base station needs to significantly reduce the transmit power to the primary user when protecting a more vulnerable victim user located within its coverage.
Figure 8 also shows that the accuracy to define $\tilde{h}^{v c}$ is not so critical when $d_{\text {norm }}$ ranges between 0.5 and 0.75 , decreasing at most $20 \%$ of the data rate of the victim celledge user. To achieve the maximum performance for the victim user, from here on, we assume a victim user path loss at a distance $d_{\text {norm }}=0.5$.

\section{Complexity reduction of NF-IWF}

Since the channel conditions can change rapidly in a wireless environment, it is crucial from a practical point of view that the power allocation computation be performed with a small number of iterations. However, Equation 7 does not have a closed form since $P_{k}^{N F, c}$ depends on $s_{k}^{c}$. Therefore, we propose an approximation of the penalty factor that reduces the complexity of Algorithm 1 by eliminating the loop of line 11 .

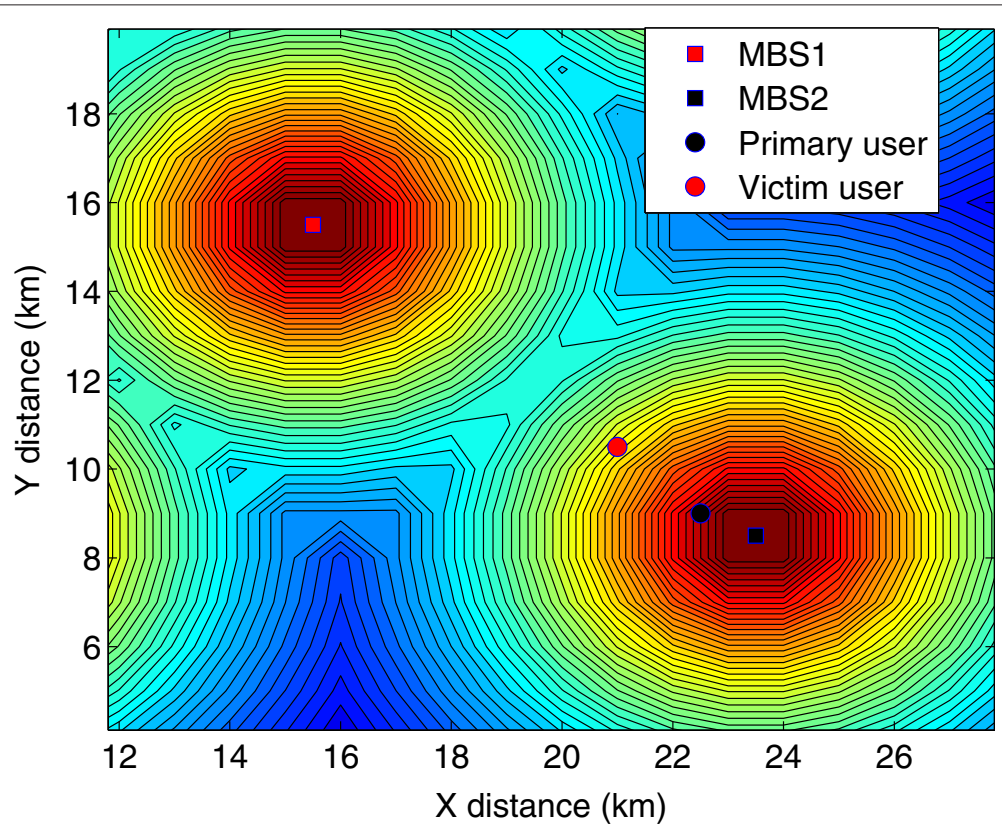

Figure 6 Case 1: high interference. The victim user is located within the coverage of the neighboring cell, resulting in high interference. 


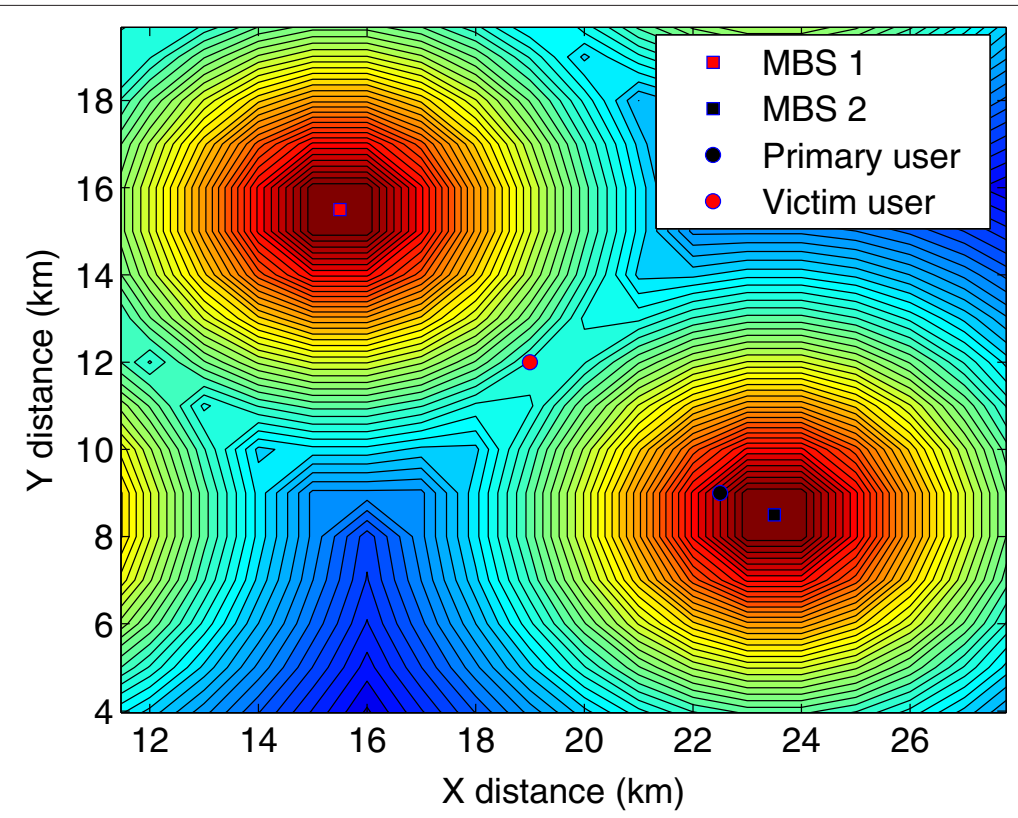

Figure 7 Case 2: low interference. The victim user is located between two cells, resulting in low interference.

Let us first expand expression (4) into

$$
\begin{array}{r}
\underset{s_{k}^{c} \forall k}{\operatorname{maximize}} w^{c} f_{s} \sum_{k} \log _{2}\left(1+\frac{1}{\Gamma} \frac{\left|h_{k}^{c}\right|^{2} s_{k}^{c}}{\sum_{\substack{\bar{c} \neq c \\
\bar{c} \in \mathcal{C}}}\left|h_{k}^{\bar{c}}\right|^{2} s_{k}^{\bar{c}}+\sigma_{k}^{c}}\right) \\
+w^{\nu c} f_{s} \sum_{k} \log _{2}\left(1+\frac{1}{\Gamma} \frac{\left|h_{k}^{\nu c}\right|^{2} s_{k}^{\nu c}}{\left|h_{k}^{\nu c, c}\right|^{2} s_{k}^{c}+\sigma_{k}^{\nu c}}\right)
\end{array}
$$$$
\text { s.t. } \quad \sum_{k} s_{k}^{c} \leq P^{c, \text { tot }}
$$$$
0 \leq s_{k}^{c} \leq s_{k}^{c \text {,mask }} \forall k \in \mathcal{K}
$$

(9)
We approximate (9) by linearizing the second term in the objective function such that the maximization problem becomes

$$
\begin{array}{cc}
\underset{s_{k}^{c} \forall k}{\operatorname{maximize}} & w^{c} f_{s} \sum_{k} \log _{2}\left(1+\frac{1}{\Gamma} \frac{\left|h_{k}^{c}\right|^{2} s_{k}^{c}}{\sum_{\substack{\bar{c} \neq c \\
\bar{c} \in \mathcal{C}}}\left|h_{k}^{\bar{c}}\right|^{2} s_{k}^{\bar{c}}+\sigma_{k}^{c}}\right) \\
& -w^{v c} f_{s} \sum_{k}\left|h_{k}^{v c, c}\right|^{2} s_{k}^{c} \\
\text { s.t. } \quad & \sum_{k} s_{k}^{c} \leq P^{c \text {,tot }} \\
& 0 \leq s_{k}^{c} \leq s_{k}^{c \text {,mask }} \forall k \in \mathcal{K}
\end{array}
$$

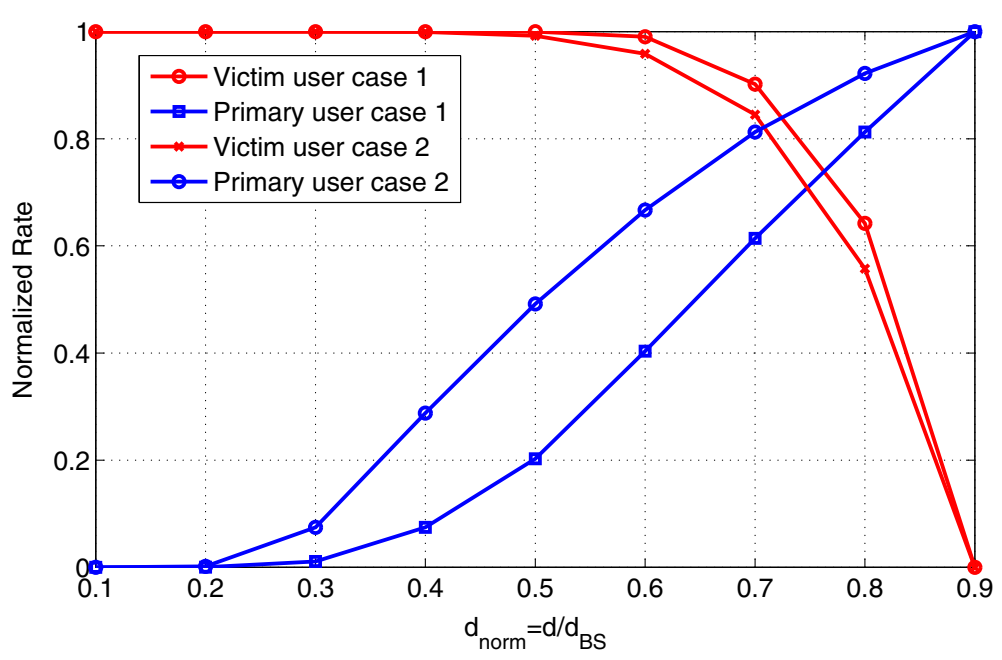

Figure 8 Normalized data rates for different values of distance $d$ when applying NF-IWF. 
For a linear equation of type $a x+b, b$ is disregarded when applying the KKT conditions, while the slope $a$ is included in $w^{v c}$ when tuning a particular operating point. The KKT stationarity condition of (10) leads to

$$
\begin{array}{r}
\forall k: \frac{w^{n, c} f_{s}\left|h_{k}^{c}\right|^{2}}{\log (2)\left(\left|h_{k}^{c}\right|^{2} s_{k}^{c}+\sum_{\bar{c} \neq c} \Gamma\left|h_{k}^{\bar{c}}\right|^{2} s_{k}^{\bar{c}}+\Gamma \sigma_{k}^{c}\right)} \\
-w^{\nu c} f_{s}\left(\left|h_{k}^{\nu c, c}\right|^{2}\right)-\lambda_{c}=0
\end{array}
$$

resulting in

$$
s_{k}^{c}=\left[\frac{\frac{w^{c} f_{s}}{\log (2)}}{\lambda_{c}+\tilde{P}_{k}^{N F, c}}-\frac{\sum_{\bar{c} \neq c} \Gamma\left|h_{k}^{\bar{c}}\right|^{2} s_{k}^{\bar{c}}+\Gamma \sigma_{k}^{c}}{\left|h_{k}^{c}\right|^{2}}\right]_{0}^{s_{k}^{c, \text { mask }}}
$$

where the penalty factor is approximated by

$$
\tilde{P}_{k}^{N F, c}=w^{\nu c} f_{s}\left|h_{k}^{v c, c}\right|^{2} .
$$

This corresponds to a linear penalty on the power level determined by the interfering channel of the victim users. In other words, the power allocation is proportionally penalized in those subcarriers with a strong interfering channel because this can easily damage the victim user data rate. This approximation results in a closed-form formula with complexity similar to IWF (and lower than NF-IWF) but improved performance as we will see later. We refer to it as NF-IWF-approx.

\section{Performance evaluation}

We consider a wireless OFDM network with the parameters shown in Table 1 . In order to evaluate the performance of our approaches, we first focus on two users (one primary user and one victim user) connected to neighboring cells. We consider two scenarios, one with interference between macrocells and one with interference between a femtocell and a macrocell. For each scenario, we analyze two cases, one with a high and another with a low interference.

We compare our proposed approaches against EPA and IWF by generating the achievable rate region, which shows the trade-off between the two user data rates. In the case of NF-IWF and NF-IWF-approx, changing the weights $w^{c}$ and $w^{v c}$ allows us to reach different full-power operating points to form the achievable rate region. This is not possible with IWF, for which the total power budget has to be tuned between 0 and $P^{c \text {,tot }}$ to obtain different operating points. This controllable tunability can be seen as an important advantage of NF-IWF.

We also compare our proposed approaches with a practical scheme like SFR, in which we divide the total bandwidth into two non-overlapping frequency bands. In order
Table 1 Simulation parameters

\begin{tabular}{lc}
\hline Parameter & Value \\
\hline System bandwidth & $5 \mathrm{MHz}$ \\
Number of subcarriers & 200 \\
$\Gamma$ & 1 \\
$\delta$ & $10^{-6}$ \\
$\gamma$ & $10^{-6}$ \\
$\Lambda^{\text {max }}$ & $10^{8}$ \\
$f_{S}$ & $2.8 \mathrm{Gsymbols} / \mathrm{s}$ \\
Channel profile & $3 \mathrm{GPP}$ SCM suburban macro \\
Macrocell total transmit power & $43 \mathrm{dBm}$ \\
Femtocell total transmit power & $15 \mathrm{dBm}$ \\
\hline
\end{tabular}

to achieve different operating points, we need to vary parameters $\alpha$ and $\beta$ from Figure 1. In the case of a multiuser scenario, each band is assigned to either primary or victim users and has a constant power level. However, in the case of two users connected to neighboring cells, the optimal approach consists in transmitting with full power to each user in one non-overlapping band, i.e., in each cell $\alpha=1$ and $\beta$ varies from 0 to 1 to achieve different operating points (discontiguous bands are not considered). In the multi-user case, this results in starvation of resources for some users.

As an upper bound, we consider the case in which the base station has full knowledge of the interfering and direct channels and of the power allocation of the neighboring base station. We call this approach NF-full, which is equivalent to the distributed algorithm (DSB) of Tsiaflakis et al. [22]. Evidently, this is not an autonomous approach, since it requires coordination between base stations.

For all the evaluated approaches, the transmit powers are updated alternately between base stations until network convergence is reached. In the following simulations, only three to five iterations are necessary for convergence.

\section{Macro-macro interference}

The two cases to be analyzed for the macro-macro scenario are shown in Figures 6 and 7. In case 1, the victim user is located within the coverage of the neighboring cell, resulting in a high interference. In case 2, the interference to the victim user is low as it is located between the coverage of the two cells.

Figure 9 shows the gains in the rate region that can be achieved with NF-IWF in the case of a high interference. For example, with a primary user data rate of $600 \mathrm{Mbps}$, NF-IWF can achieve $6 \mathrm{Mbps}$ for the victim user instead of 1.7 Mbps for IWF, 0.4 Mbps for SFR, and 0.1 Mbps for EPA, i.e., an increase by a factor of 3.5 compared to 


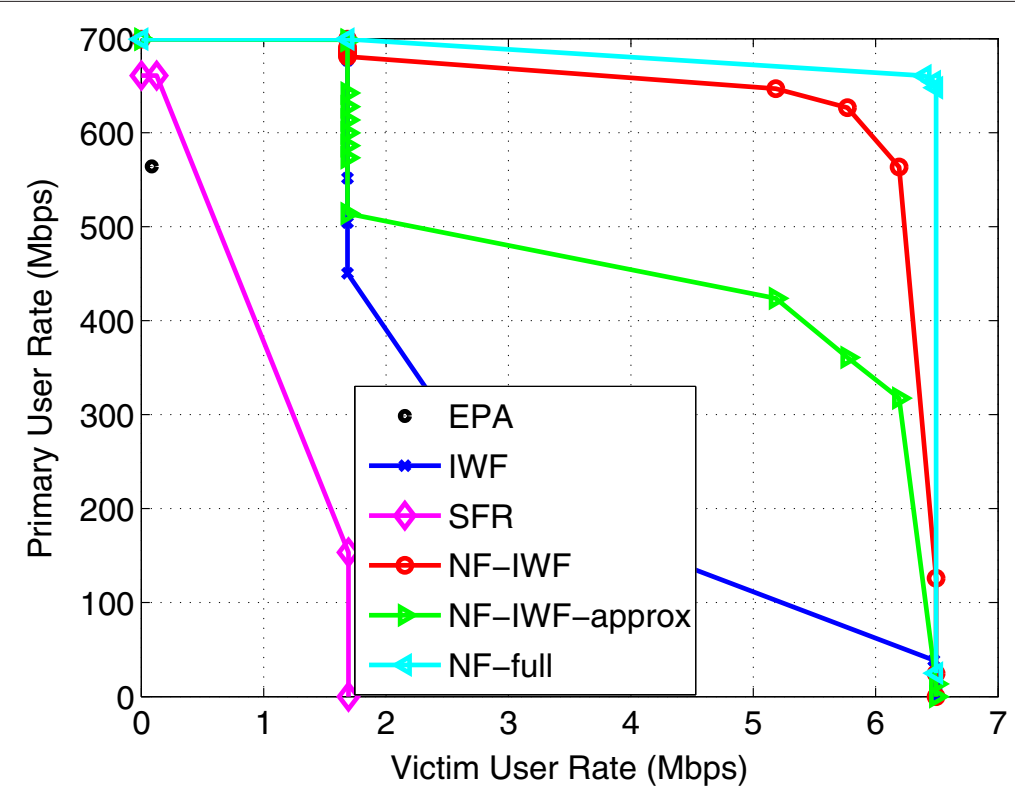

Figure 9 Rate region of the primary and victim users of the macro-macro high interference case.

IWF, a factor of 15 compared to SFR, and a factor of 60 compared to EPA. Furthermore, the difference between the approach with full knowledge and NF-IWF proves to be marginal. NF-IWF-approx can achieve similar gains with reduced complexity when the data rate of the primary user is lower than 500 Mbps. Needless to say, the underperformance and lack of tunability of EPA are evident. SFR is able to improve the performance for both primary and victim users compared to EPA, but not compared to IWF. This is because SFR partitions the available bandwidth in non-overlapping bands, avoiding interference in this way. However, SFR does not exploit a variable per-subcarrier power allocation, which results in a large degradation.

Figure 10 shows the rate region for the low interference case. Except for EPA and SFR, the performance of all algorithms is similar. This is because the direct channels are stronger compared to the interfering channels; hence, even a greedy algorithm like IWF has improved performance.

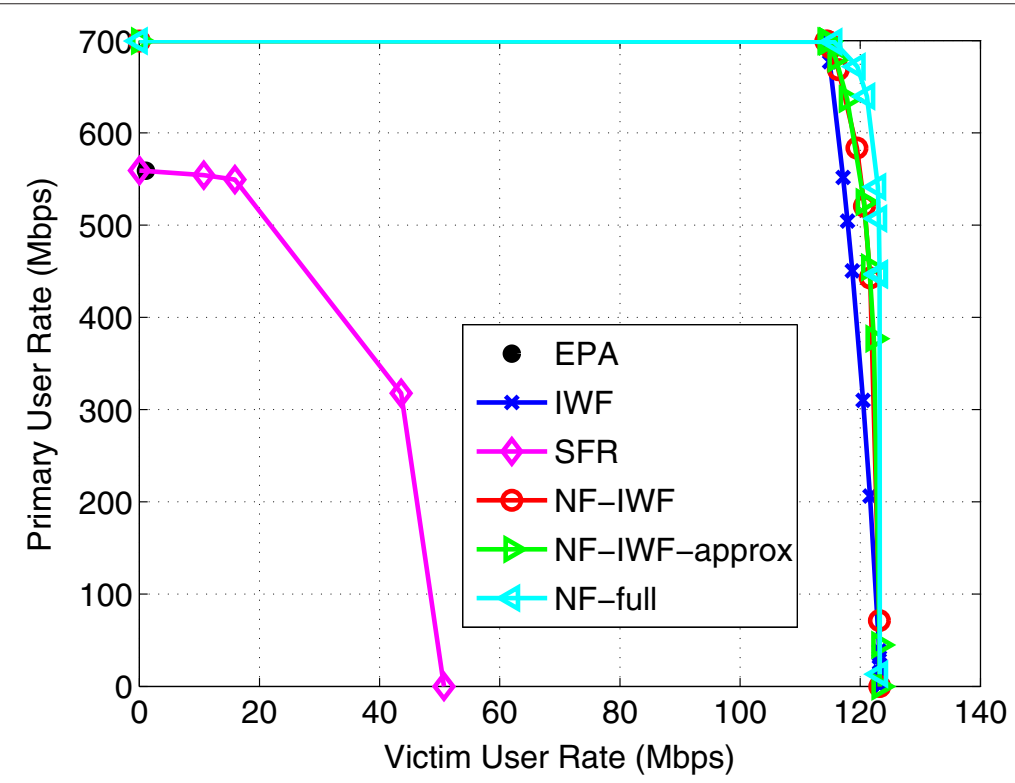

Figure 10 Rate region of the primary and victim users of the macro-macro low interference case. 


\section{Femto-macro interference}

The two cases to be analyzed for the scenario with femtomacro interference are shown in Figures 11 and 12. In case 1 , the victim user of the macrocell is located within the coverage of the neighboring femtocell, resulting in a high interference. In case 2 , the interference to the victim user is low as it is located far from the femtocell coverage.

Figure 13 shows that with a primary user data rate of $80 \mathrm{Mbps}$, NF-IWF can achieve 1.45 Mbps for the victim user instead of 0.5 Mbps for IWF, 0.7 for SFR, and 0.02 Mbps for EPA, i.e., an increase by a factor of 2.9 compared to IWF, a factor of 2 compared to SFR, and a factor of 73 compared to EPA. For this primary user data rate, NF-IWF-approx achieves the same gains. In the low interference case, all the algorithms perform similar except for EPA and SFR as seen in Figure 14.

It is interesting to note that with a high interference, NF-IWF-approx provided more than a $50 \%$ increase in the victim user data rate compared to IWF with less complexity than NF-IWF. This result indicates that the linear approximation of the penalty factor indeed provides most of the useful information to achieve significant gains.

\section{Multi-user multi-cell interference}

The achievable rate regions presented so far show the advantages of our proposed approaches in a two-user evaluation. However, a multi-user multi-cell evaluation is needed to assess the total network gains.
We consider a wireless heterogeneous network consisting of 5 macrocells (evenly distributed), 20 femtocells (randomly distributed), and 500 users (randomly distributed). Any two neighboring cells share a set of subcarriers and each user is randomly allocated the same number of consecutive subcarriers within a cell. This topology is shown in Figure 3, and the performance results are shown in Figure 15. The user color indicates the base station to which the users are attached. Users located within the coverage of their macrocell are classified as macro primary users, while users located farther than the coverage of their macrocell (i.e., closer to a neighboring cell than to the cell they are attached to) are classified as macro victim users and are those to be protected. Users attached to femtocells are classified as femto users.

For a fair comparison, all base stations use the same algorithm with the best rate region operating point for any two neighboring cells. For IWF, the best operating point corresponds to a full-power scheme, while for SFR, NFIWF, NF-IWF-approx, and NF-full, we consider a point on the rate region boundary with a tangent of slope equal to -1 .

From the cumulative distribution function (CDF) of Figure 15, we can observe the gains achieved by our proposed approaches. As expected, NF-full achieves the best data rate performance for the macro victim users, followed closely by NF-IWF and NF-IWF-approx. IWF and EPA present a clear underperformance due to their greediness towards victim users, while the SFR suffers from the band partitioning and lack of power control within

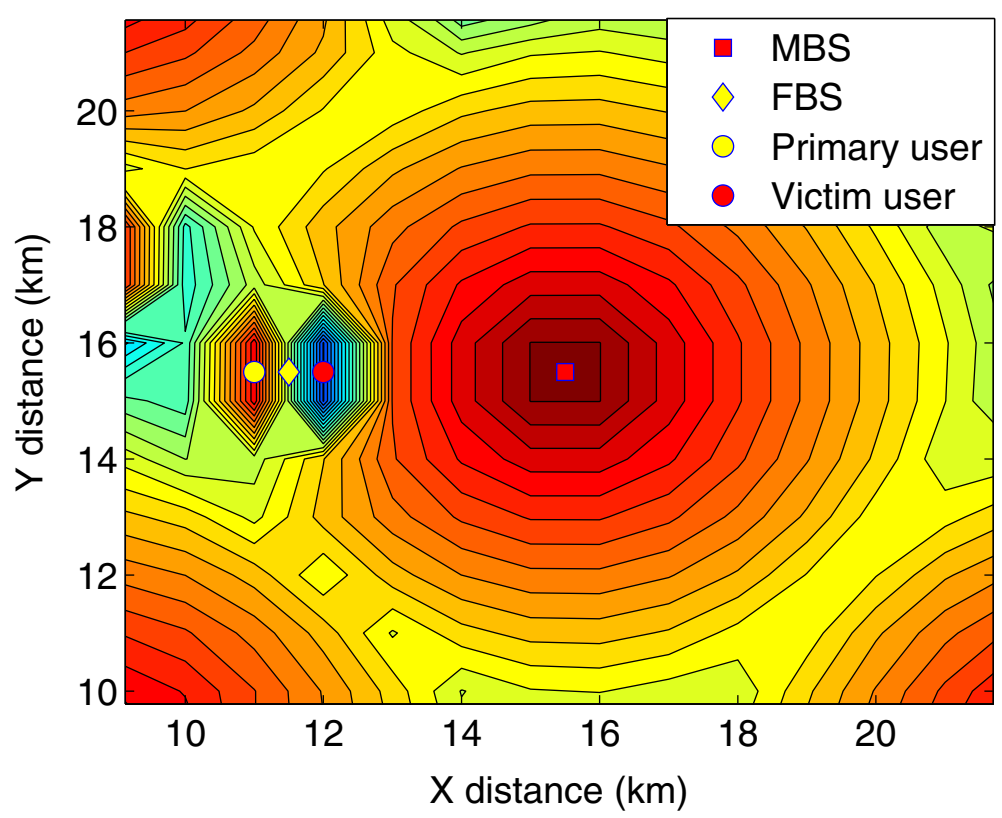

Figure 11 Case 1: high interference. The victim user attached to the macrocell is located within the coverage of the neighboring femtocell, resulting in a high interference. 


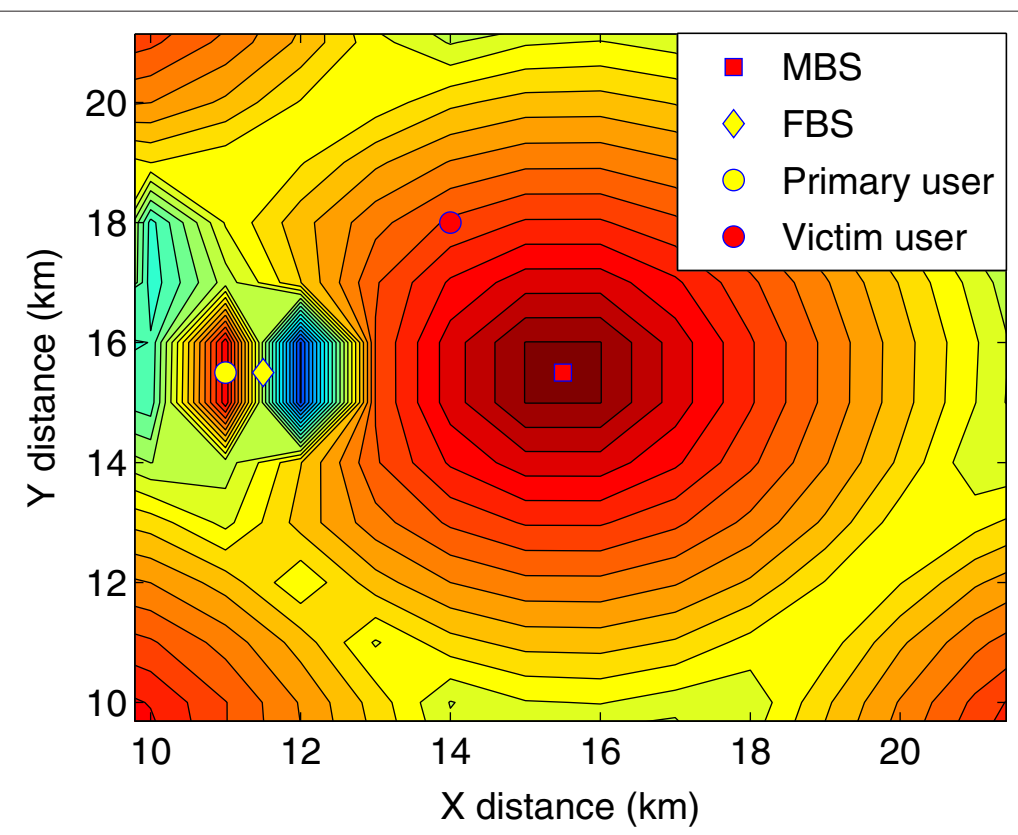

Figure 12 Case 2: low interference. The victim user attached to the macrocell is far from the femtocell, resulting in a low interference.

each band. For example, with the NF algorithms, macro victim users have a very large probability of obtaining a normalized data rate higher than 95\%; while with SFR, IWF, and EPA, they have $40 \%$ probability of a normalized data rate lower than $70 \%, 50 \%$, and $5 \%$, respectively. Femto users achieve similar gains.

Additionally, the gains come with a marginal degradation in the macro primary user data rates. Since we consider the full-power operating point for IWF, it achieves the highest data rate for the macro primary users, followed closely by NF-full, NF-IWF, NF-IWF-approx, and SFR. EPA can sometimes present a higher primary user data rate compared to SFR and NF algorithms. This is because, depending on the channel conditions, the EPA operating point can have a higher primary user data rate than the operating point of SFR and NF algorithms.

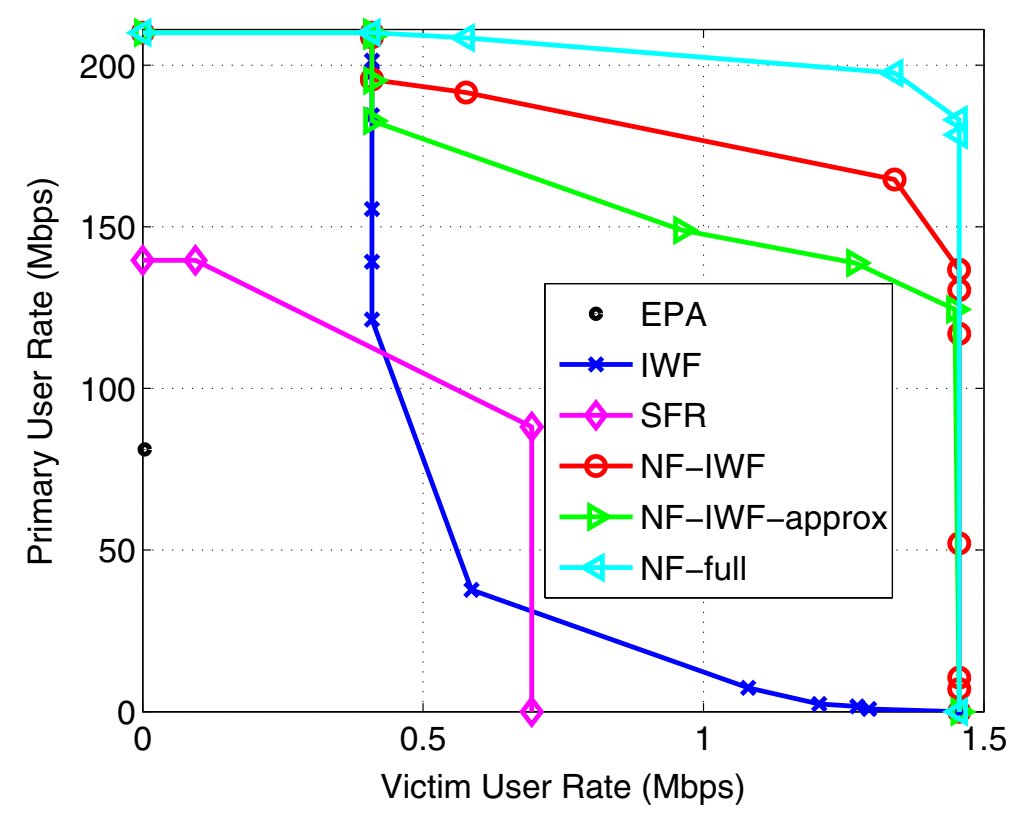

Figure 13 Rate region of the primary and victim users of the macro-femto high interference case. 


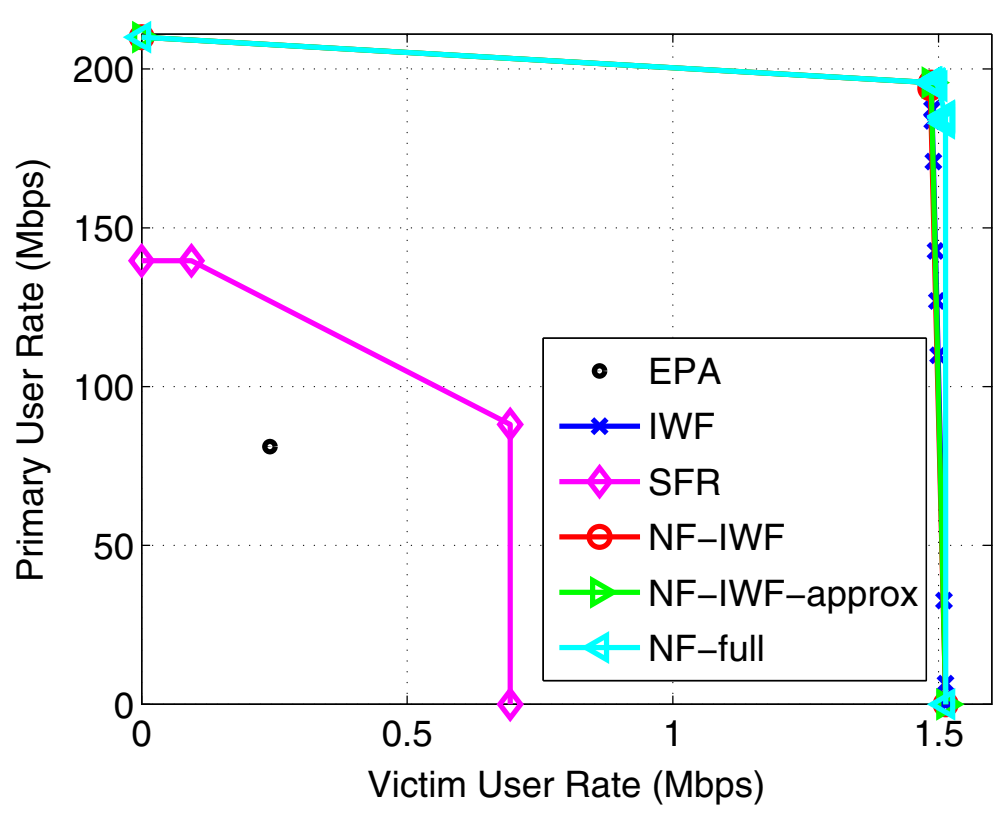

Figure 14 Rate region of the primary and victim users of the macro-femto low interference case.
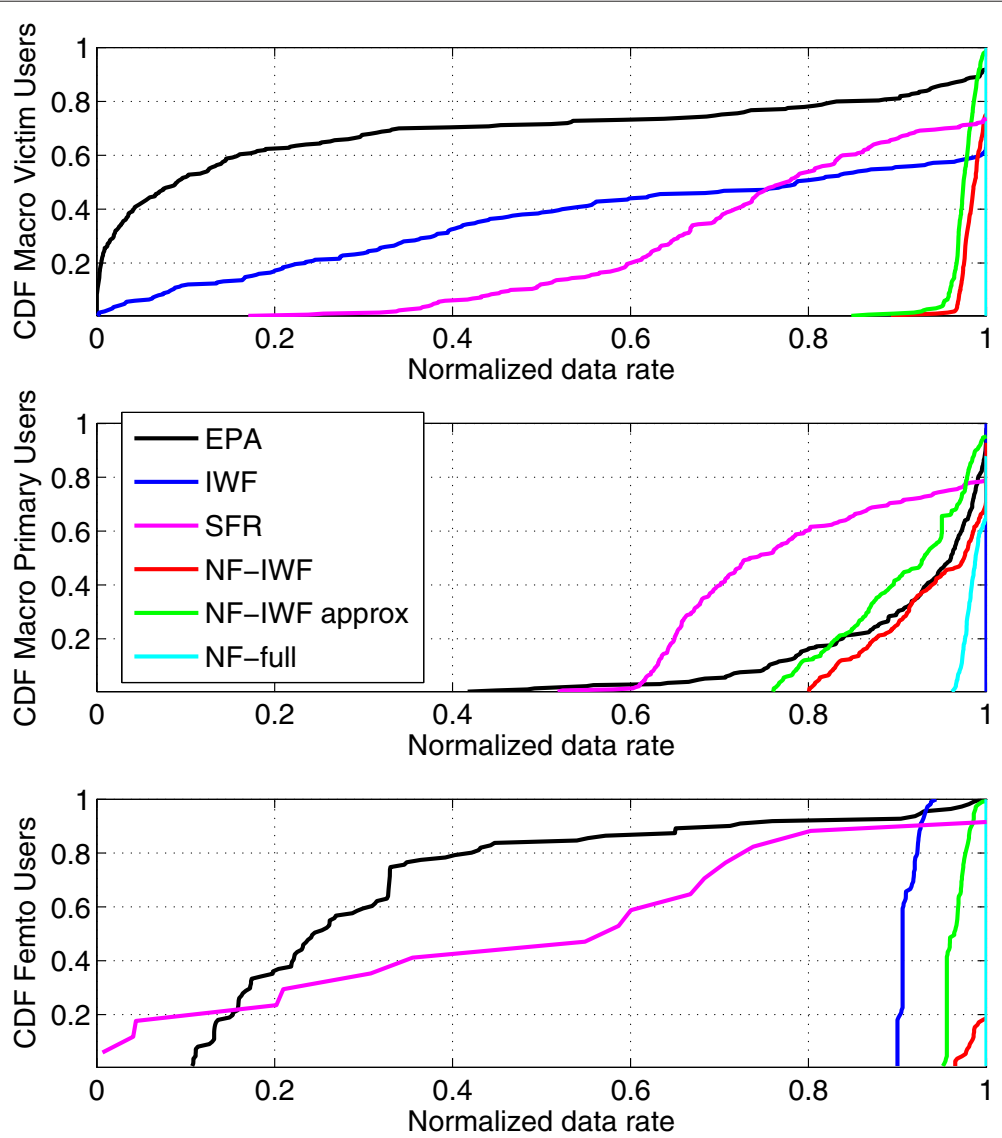

Figure 15 CDF of victim and primary users of the multi-user scenario. 
However, the NF algorithms never achieve a normalized data rate lower than $75 \%$.

\section{Sensitivity analysis}

In this section, we evaluate the data rate performance of our approaches for a given channel estimation error in all the channels with some frequency variation, i.e., $h_{k}^{c}, h_{k}^{\bar{c}}$, and $h_{k}^{v c, c}$. We model the channel estimation error $\Delta$ as a Gaussian random variable with zero mean and different values of standard deviation (std). A visual representation of the channel estimation error is shown in Figure 16. The value of $s t d$ represents a fraction of the average channel value.

The power allocation is then computed as

$$
\hat{s}_{k}^{c}=\left[\frac{\frac{w^{c} f_{s}}{\log (2)}}{\lambda_{c}+\hat{P}_{k}^{N F, c}}-\frac{\sum_{\bar{c} \neq c} \Gamma\left|\hat{h}_{k}^{\bar{c}}\right|^{2} s_{k}^{\bar{c}}+\Gamma \sigma_{k}^{c}}{\left|\hat{h}_{k}^{c}\right|^{2}}\right]_{0}^{s_{k}^{c, \text { mask }}}
$$

with the penalty factor of NF-IWF modeled as

$$
\begin{aligned}
& \hat{P}_{k}^{N F, c} \\
& =\frac{\left.w^{v c} f_{s} \tilde{h}^{v c}\right|^{2} s_{k}^{v c} \Gamma\left|\hat{h}_{k}^{v c, c}\right|^{2}}{\log (2)\left(\Gamma\left|\hat{h}_{k}^{v c, c}\right|^{2} s_{k}^{c}+\Gamma \sigma_{k}^{v c}\right)\left(\left|\tilde{h}^{v c}\right|^{2} s_{k}^{v c}+\Gamma\left|\hat{h}_{k}^{v c, c}\right|^{2} s_{k}^{c}+\Gamma \sigma_{k}^{v c}\right)}
\end{aligned}
$$

and the penalty factor of NF-IWF-approx modeled as

$$
\hat{\tilde{P}}_{k}^{N F, c}=w^{\nu c} f_{s}\left|\hat{h}_{k}^{v c, c}\right|^{2}
$$

where $\hat{h}=h+\Delta$ for all channels, with $h$ being the true channel (subscripts and superscripts omitted).

With the obtained power allocation values, we compute the achievable data rate for each cell as

$$
R^{c}=f_{s} \sum_{k \in \mathcal{K}} \log _{2}\left(1+\frac{1}{\Gamma} \frac{\left|h_{k}^{c}\right|^{2} \hat{s}_{k}^{c}}{\left.\sum_{\substack{\bar{c} \neq c \\ \bar{c} \in \mathcal{C}}}\left|h_{k}^{\bar{c}}\right|^{2}\right|_{k} ^{\bar{c}}+\sigma_{k}^{c}}\right) .
$$

Figure 17 shows that our approaches are robust to very high channel estimation errors and can still present significant gains compared to IWF even with $s t d=1$. It is also interesting to note that for small values of $s t d$, the primary user data rate can improve compared to the case without errors. This is because the primary user is less vulnerable to channel estimation errors and can profit from the power level decrease of the victim user.

\section{Computational complexity for convergence}

In this section, we quantify the computational complexity needed for each algorithm to converge to transmit powers satisfying the total power constraints. EPA and SFR do not need to update the transmit powers. All the NF algorithms have two or three levels of iterations as shown in Algorithm 1. First, there is an outer level that iterates over the base stations until network convergence in the power allocation is achieved. The number of iterations at this level is denoted with $N_{o}$. A second level of iterations is used for the bisection search of $\lambda_{c}$ in each base station until the total power constraint is satisfied. The number of

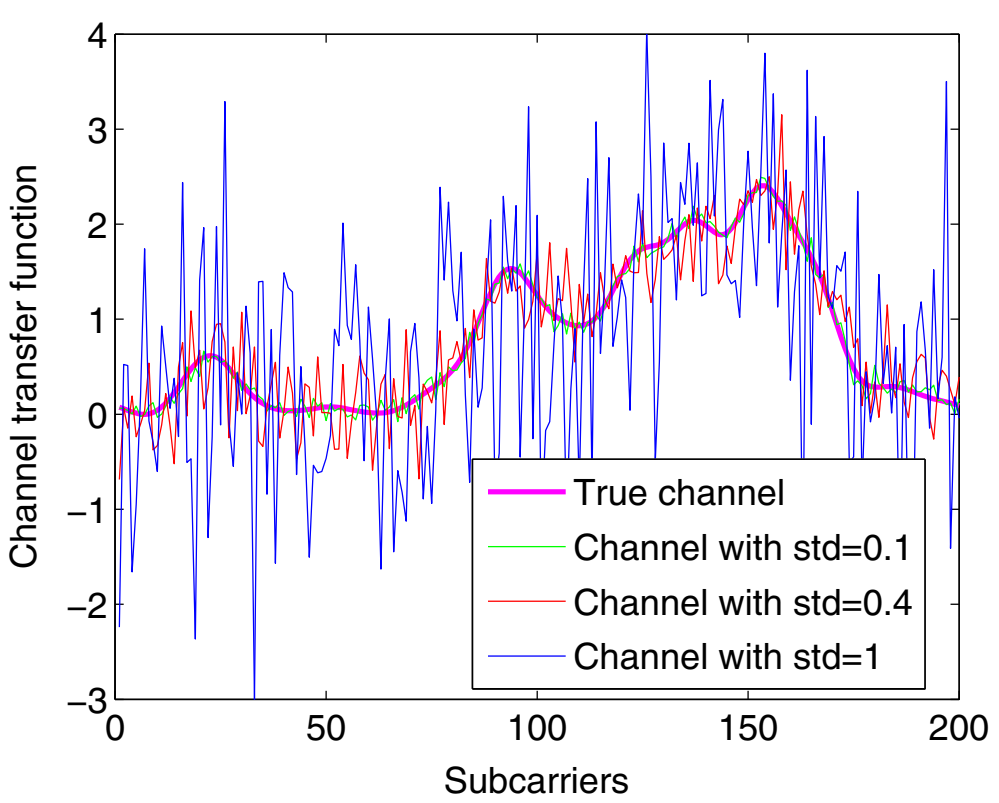

Figure 16 Channel transfer function with an estimation error of zero mean and standard deviation (std). 


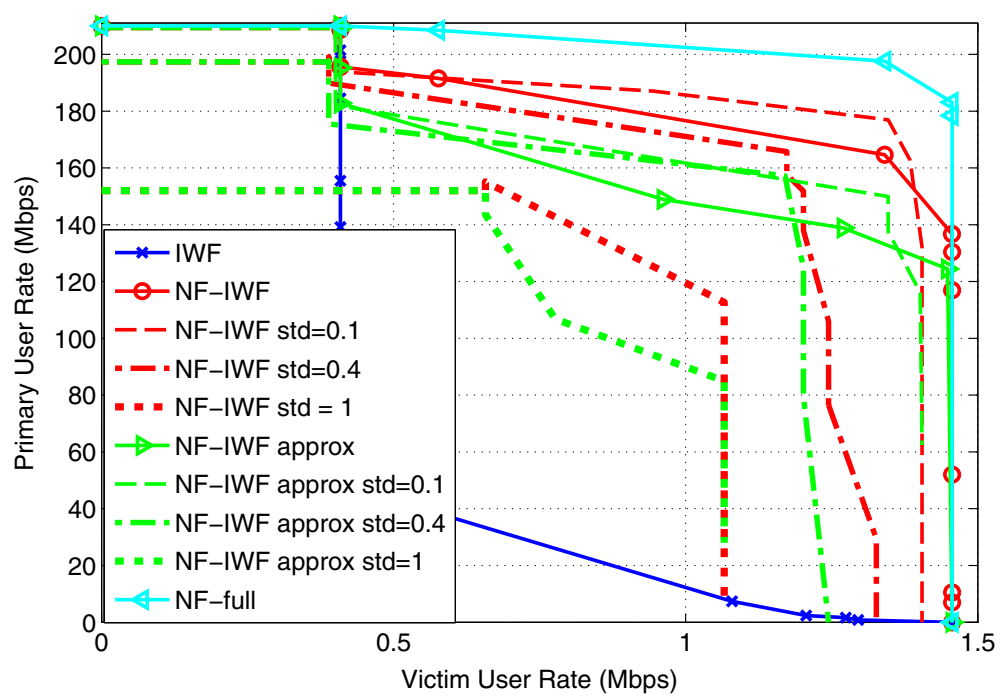

Figure 17 Sensitivity analysis on the channel estimation.

iterations at this level is denoted by $N_{\lambda}$. These two levels of iterations are shared by IWF, NF-IWF, and NF-IWFapprox. However, NF-IWF has an extra level of iterations on $P_{k}^{N F, c}$ to update $s_{k}^{c}$. The number of iterations at this level is denoted with $N_{P}$.

The convergence properties of the proposed algorithms can be seen in Figure 18 in terms of the number of iterations per subcarrier per base station, i.e., we evaluate the product $N_{\lambda} N_{P}$. We can observe that after ten iterations, all of the proposed algorithms reach convergence. However, NF-IWF-approx is the first one to reach convergence at three iterations within a $10 \%$ range of the final value, followed by NF-IWF at four iterations, and finally NF-full at six iterations. Since we consider the best operating point in the rate region of each algorithm, the different algorithms converge to a different value. Interesting to note is that both NF-IWF and NF-IWF-approx show a fluctuating behavior during the first iterations, while NF-full shows a smoother behavior. This is due to the approximations involved in NF-IWF and NF-IWFapprox that make the algorithms fluctuate before reaching convergence.

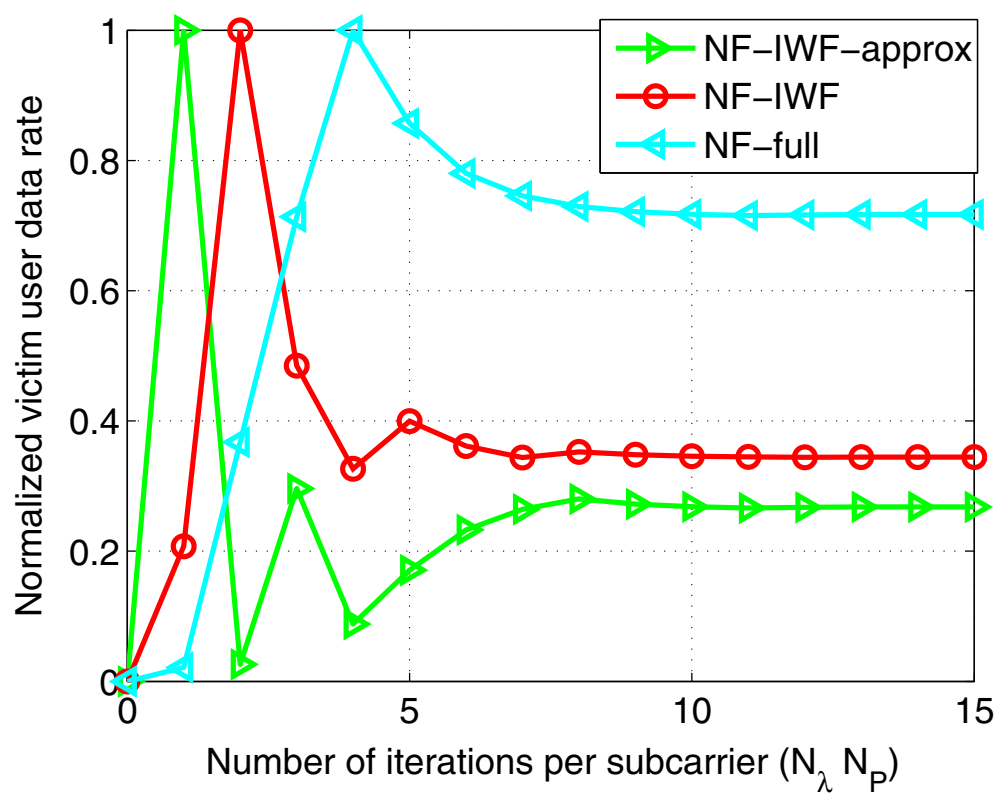

Figure 18 Number of iterations per subcarrier per base station to reach convergence in the proposed algorithms. 


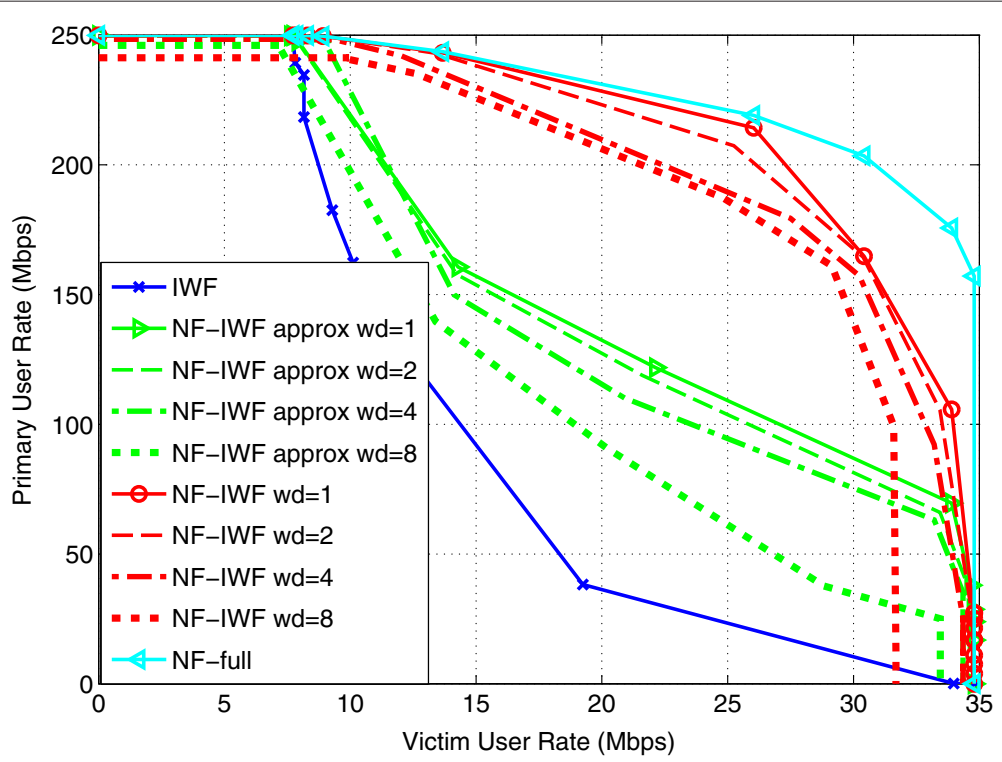

Figure 19 Complexity analysis for different values of windows.

Within the different levels of iterations, the transmit power computation needs to be done on a per-subcarrier basis, i.e., we need $\mathcal{K}$ transmit power calculations. This leads to a total complexity in the number of power computations of $N_{o} N_{\lambda} N_{P} \mathcal{K}$ for NF-IWF and $N_{o} N_{\lambda} \mathcal{K}$ for IWF and NF-IWF-approx.

This complexity can be further reduced by exploiting the frequency correlation of the wireless channel. We analyze the impact of this complexity reduction for both NF-IWF and NF-IWF-approx. For this purpose, we define a window of $w d$ subcarriers in which we reuse the transmit power value of one of the middle subcarriers. The data rate impact of this reduction can be seen in Figure 19. We note that the degradation is marginal for a window of two and four subcarriers and becomes more visible for a window of eight subcarriers. A similar rationale can be used to exploit the time correlation of the channel.

\section{Conclusions}

In this paper, we have proposed NF-IWF, a neighborfriendly autonomous algorithm for power control in wireless heterogeneous networks. In contrast to greedy algorithms like EPA and IWF, NF-IWF protects the data rate of victim users located within a certain distance from the base station. This is done through a factor that penalizes the power allocation in those subcarriers that damage the data rate of victim users. Furthermore, we have developed a low-complexity version, NF-IWF-approx, which reduces the complexity by assuming a linear approximation of the victim user data rate, resulting in a closedform formula. Other options have been analyzed to further reduce complexity by exploiting frequency and time correlation of the channel. We have evaluated our algorithms under high and low interference conditions in a heterogeneous network with macro- and femtocells. Our approaches present higher tunability, robustness, and better data rate performance, especially in high interference conditions. We have shown an increase by a factor of 3.5 compared to IWF, 15 compared to SFR, and 60 compared to EPA in the victim user data rate with a marginal decrease of the primary user data rate. We have also shown that the rate region of our approaches lies close to the upper bound with full knowledge of the interfering channel and the power allocation of the neighboring cell.

\section{Competing interests}

The authors declare that they have no competing interests.

\section{Acknowledgments}

This research work was carried out at the ESAT Laboratory of KU Leuven, in the frame of KU Leuven Research Council PFV/10/002 (OPTEC), Concerted Research Action GOA-MaNet, FWO project G091213N 'Cross-layer optimization with real-time adaptive dynamic spectrum management for fourth generation broadband access networks', and the Belgian Programme on Interuniversity Attraction Poles initiated by the Belgian Federal Science Policy Office: IUAP 'Belgian network on Stochastic modelling, analysis, design and optimization of communication systems' (BESTCOM) 2012-2017. The scientific responsibility is assumed by its authors. The first author acknowledges the support of the Mexican National Council for Science and Technology (CONACYT). Part of this work has been submitted for presentation at the 11th International Symposium on Wireless Communication Systems (ISWCS), Barcelona, August, 2014

\section{Author details}

${ }^{1}$ STADIUS Center for Dynamical Systems, Signal Processing and Data Analytics, Kasteelpark Arenberg 10, 3000 Leuven, Belgium. ${ }^{2}$ Bell Labs, Alcatel-Lucent, Copernicuslaan 50, B-2018 Antwerp, Belgium. ${ }^{3}$ Université Catholique de Louvain (UCL), ICTEAM Institute, Digital Communications Group, Place du Levant 2, 1348 Louvain-la-Neuve, Belgium.

Received: 19 June 2014 Accepted: 17 October 2014 Published: 24 October 2014 


\section{References}

1. W Webb, The european spectrum capacity crunch. [Online; accessed 6-March-2013], Available: http://www.microwavejournal.com/articles/ 18176-the-european-spectrum-capacity-crunch, Sep. 2012

2. O Chapman, Small cell vendors race to fulfill operator demand for LTE. [Online; accessed 11-September-2013], Available: http://www. smallcellforum.org/newsstory-small-cell-vendors-race-to-fulfilloperator-demand-for-Ite, Feb. 2012

3. 3GPP, Small cell forum - new name for 3GPP partner. [Online; accessed 6-March-2013], Available: http://www.3gpp.org/Small-Cell-Forum-newname-for-3GPP, Feb. 2012

4. Small Cell Forum. [Online; accessed 6-March-2013], Available: http://www.smallcellforum.org, Feb. 2013

5. C Kosta, B Hunt, A UI Quddus, R Tafazolli, On interference avoidance through inter-cell interference coordination (ICIC) based on OFDMA mobile systems. IEEE Commun. Surv. Tutor. 15(3), 1-23 (2013)

6. TWang, L Vandendorpe, Iterative resource allocation for maximizing weighted sum min-rate in downlink cellular OFDMA systems. IEEE Trans. Signal Process. 59(1), 223-234 (2011)

7. D Lopez-Perez, I Guvenc, Roche de la G, M Kountouris, TQS Quek, J Zhang, Enhanced inter-cell interference coordination challenges in heterogeneous networks. IEEE Wireless Commun. 18(3), 22-30 (2011)

8. R Kwan, C Leung, A survey of scheduling and interference mitigation in LTE. J. Electrical Comput. Eng. 2010, 1-10 (2010)

9. G Boudreau, J Panicker, N Guo, R Chang, N Wang, S Vrzic, Interference coordination and cancellation for $4 \mathrm{G}$ networks. IEEE Commun. Mag. 47(4), 74-81 (2009)

10. M Necker, Interference coordination in cellular OFDMA networks. IEEE Netw. 22(6), 12-19 (2008)

11. 3GPP, Soft frequency reuse scheme for UTRAN LTE. [Online; accessed 6-March-2013], Available: ftp://ftp.3gpp.org/tsg_ran/WG1_RL1/ TSGR1_41/Docs/R1-050507.zip, R1-050507, Athens, Greece, May 2005

12. D Gonzalez G, M Garcia-Lozano, S Ruiz Boque, D Seop Lee, Optimization of soft frequency reuse for irregular LTE macrocellular networks. IEEE Trans. Wireless Commun. 12(5), 2410-2423 (2013)

13. S Oh, H Kim, B Ryu, N Park, in International Conference on Compute Communications and Networks (ICCCN). Inbound mobility management on LTE-advanced femtocell topology using X2 interface (Maui, Hawaii, 2011)

14. W Liu, C Hu, D Wei, M Peng, W Wang, in IEEE International Conference on Broadband Network and Multimedia Technology (IC-BNMT). An overload indicator \& high interference indicator hybrid scheme for inter-cell interference coordination in LTE system (Beijing, China, 2010), pp. 514-518

15. R Combes, Z Altman, M Haddad, E Altman, in IEEE international conference on communications workshops (ICC). Self-optimizing strategies for interference coordination in OFDMA networks (Kyoto, Japan, 2011)

16. M Rahman, H Yanikomeroglu, W Wong, in IEEE Wireless Communications and Networking Conference (WCNC). Interference avoidance with dynamic inter-cell coordination for downlink LTE system (Budapest, Hungary, 2009)

17. G Fodor, C Koutsimanis, A Racz, N Reider, A Simonsson, W Muller, Intercell interference coordination in OFDMA networks and in the 3GPP long term evolution system. Journal of Commun. 4(7), 445-453 (2002)

18. 3GPP, Evolved universal terrestrial radio access (EUTRA); overall description; stage 2 (release 11). [Online; accessed 6-March-2013], Available: http://www.3gpp.org/ftp/Specs/archive/36_series/36.300/ 36300-b10.zip, TS 36.300 V11.1.0, Mar. 2012

19. 3GPP, Physical layer procedures. [Online; accessed 6-March-2013], Available: http://www.3gpp.org/ftp/Specs/archive/36_series/36.213/ 36213-a20.zip, TS 36.213 V10.2.0, Mar. 2012

20. W Yu, G Ginis, J Cioffi, Distributed multiuser power control for digita subscriber lines. IEEE Trans. Selected Areas Commun. 20(5), 1105-1115 (2002)

21. R Cendrillon, J Huang, M Chiang, M Moonen, Autonomous spectrum balancing for digital subscriber lines. IEEE Trans. Signal Process. 55(8), 4241-4257 (2007)

22. P Tsiaflakis, M Diehl, M Moonen, Distributed spectrum management algorithms for multiuser DSL networks. IEEE Trans. Signal Process. 56(2), 4825-4843 (2008)

23. K Son, S Lee, Y Yi, S Chong, REFIM: A practical interference management in heterogeneous wireless access networks. IEEE Trans. Selected Areas Commun. 29(6) (2011)
24. 3GPP, Spatial channel model for multiple input multiple output (MIMO) simulations. [Online; accessed 6-March-2013], Available: http://www. 3gpp.org/ftp/Specs/archive/25_series/25.996/25996-b00.zip, TR 25.996, v11.0.0, Sep. 2012

25. R Cendrillon, W Yu, M Moonen, J Verlinden, T Bostoen, Optimal multiuser spectrum balancing for digital subscriber lines. IEEE Trans. Commun. 54(5), 922-933 (2006)

26. 3GPP, Considerations on interference coordination in heterogeneous networks. [Online; accessed 6-March-2013], Available: http://www.3gpp org/ftp/tsg_ran/WG1_RL1/TSGR1_59b/Docs/R1-100236.zip, R1-101369, San Francisco, CA, Feb. 2010

27. 3GPP, Summary of the description of candidate eICIC solutions. [Online; accessed 6-March-2013], Available: http://www.3gpp.org/ftp/tsg_ran/ WG1_RL1/TSGR1_62/Docs/R1-105081.zip, R1-104968, Madrid, Spain, Aug 2010

28. T Yang, L Zhang, in IEEE International Symposium on Personal, Indoor, and Mobile Radio Communications. Approaches to enhancing autonomous power control at femto under co-channel deployment of macrocell and femtocell (Toronto, Canada, 2011)

doi:10.1186/1687-1499-2014-175

Cite this article as: Torrea-Duran et al.: Neighbor-friendly autonomous power control in wireless heterogeneous networks. EURASIP Journal on Wireless Communications and Networking 2014 2014:175.

\section{Submit your manuscript to a SpringerOpen ${ }^{\odot}$ journal and benefit from:}

- Convenient online submission

Rigorous peer review

- Immediate publication on acceptance

- Open access: articles freely available online

- High visibility within the field

- Retaining the copyright to your article

Submit your next manuscript at $>$ springeropen.com 